\title{
Intrinsic motivation among clinic-referred children
}

\author{
STEPHEN J. DOLLINGER and JUDITH A. SEITERS \\ Southern Illinois University, Carbondale, Illinois
}

\begin{abstract}
This study examined the reliability and validity of Harter's $(1980,1981)$ intrinsic motivation questionnaire among 50 children referred for evaluations to an interdisciplinary clinic. With one exception, the five scales proved to have acceptable internal consistency reliability. The curiosity scale's reliability was markedly lower than that reported for other samples. Factor analysis of the five scales revealed the same two-factor structure as reported in research with nonclinical samples. An intrinsic motivation-personality composite, derived from maternal reports on the California Child Q-Set (Block \& Block, 1980), provided correlational support for the validity of two scales: preference for challenge and curiosity. Moreover, significant correlations were obtained between several scales and academic retention, tested intelligence, and educational attainment of the child's father.
\end{abstract}

Although there are numerous tests for measuring the child's capabilities and achievement, there are few designed to tap the child's motivation or attitudes about learning. Those that have been designed over the years tend to be psychometrically weak or lend themselves more to research than clinical purposes. One exception to this rule is Harter's (1981) recently developed scale for measuring intrinsic versus extrinsic orientation in the classroom. On the basis of a decade of laboratory research (e.g., Harter, 1978), Harter devised the instrument to tap children's attitudes about learning, particularly in the classroom situation. The inventory partitions the child's attitudes into five areas: preference for challenging versus easy work; preference to learn out of curiosity versus extrinsic reasons (to earn high marks, to please adults); desire for independent mastery (preference to learn new material on one's own) versus dependence on teacher; preference for independent judgment about what to learn versus dependence on teacher; and presence of internal criteria for judging performance versus dependence on external signs (grades, rewards).

Research use of the inventory has proliferated in the last four years. Several researchers have used the motivation scale to study, in addition to other educational applications, creativity (Amabile, Hennessey, \& Grossman, 1986) and helplessness (Boggiano \& Barrett, 1985). Of particular relevance is a study by Lincoln and Chazan (1979), using an early version of the inventory. They found that learning-disabled youngsters scored lower than normal students on the independent judgment and inter-

J. A. Seiters is Director of the Clinical Center at Southern Illinois University, where this study was conducted and where S. Dollinger holds a parttime appointment. Thanks are extended to Diane Felton, Faith Phillips, Valerie Samuel, Brian Thomson, and Barb Tylenda for their help in data collection. Address correspondence to $S$. Dollinger at the Department of Psychology, Southern Illinois University, Carbondale, IL 62901. nal criteria scales, the two scales Harter identified as forming an "informational" factor in higher order factor analyses. Because these are also the scales that typically show the strongest age effects, one might infer that the learningdisabled group showed developmentally immature attitudes in these areas.

One general implication of the literature to date is that the inventory has promise for potential clinical application. However, more research is needed on the reliability and validity of the motivation and competence scales before they can be applied routinely to diverse clinical samples. Thus, the first purpose of the present study was to examine the psychometric properties of the intrinsic motivation inventory in a clinic sample. The second purpose was to examine the scales' construct validity in terms of whether they correlated with other presumably relevant variables-including age, intelligence, retention in school, and maternal report of personality characteristics-used to provide an alternative measure of intrinsic motivation.

Predictions can be made for most of these variables. On the basis of previous research (Harter, 1980, 1981), the independent judgment and internal criteria scales can be expected to correlate positively with chronological age, whereas the challenge, curiosity, and independent mastery scales might be expected to correlate inversely or not at all with age. If the scales are valid, positive correlations should emerge between the maternal personality description of intrinsic motivation and the inventory. Finally, negative relations might be expected between the motivation scales and academic retention if it is assumed that retention further directs a child's attention to a salient extrinsic motive-being promoted.

\section{METHOD}

\section{Subjects}

The subjects were 50 children ( 37 boys, 13 girls) between the ages of 7,3 and 13,10 (Grade 2 through Grade 7) referred to an interdiscipli- 
nary university clinic for evaluations of academic or behavioral difficulties. None were considered psychotic or retarded. Eleven had been retained for at least 1 year in school.

\section{Materials}

Harter's $(1980,1981)$ Intrinsic versus Extrinsic Orientation in the Classroom Scale includes six items for each of the five scales noted earlier. Each item is scaled from 1 to 4, with high scores reflecting more intrinsic motivation. Each scale score is the mean of the item responses for that scale. A sample item from the internal-criteria scale illustrates the structured-alternatives format of the questionnaire: "Some kids know when they've made a mistake without checking with the teacher. But other kids need to check with the teacher to know if they've made a mistake." Children are instructed to decide whether they are more like the first or second type of child and whether this is "sort of" or "really" true for them.

The Block and Block (1980) California Child Q-Set (CCQ) was given to mothers as a method for obtaining an alternative measure of the child's intrinsic motivation. It was selected because about $10 \%$ of this 100 -item set is highly relevant to the concept of intrinsic motivation. In accord with standard directions, mothers were asked to place 11 of the 100 cards into each of nine categories ranging from 1 (extremely uncharacteristic or negatively salient) to 9 (extremely characteristic or salient). A twelfth card was to be placed in the middle category (5: relatively neutral or unimportant). Statements considered as relevant included the following:

Is resourceful in initiating activities.

Likes to compete; tests and compares self against others.

Is curious and exploring; eager to learn; open to new experience.

Is persistent in activities; does not give up easily.

Has high standards of performance for self.

Tends to be indecisive and vacillating (-)

Is planful; thinks ahead.

Becomes strongly involved in what he/she does.

Is self-assertive.

Seeks to be independent and autonomous.

Is self-reliant, confident; trusts own judgment.

Looks to adults for help and direction (-).

With the exception of the last statement, the items intercorrelated in appropriate directions with respect to their meaning. A composite was derived by summing the other 11 items with unit weighting, with one item weighted negatively. This composite was internally consistent as determined by coefficient alpha (.84), and has some convergent validity in terms of its correlation with IQ scores in this sample $(r=.41$, $p<.01$ ).

Routine clinic forms were used to determine whether the child had been retained (school report) and the parent's educational level (parent report). Intelligence test scores were extracted from assessment reports, in most cases involving the Wechsler Intelligence Scale for Children-Revised.

\section{Procedure}

Before each child's testing began, his/her mother was escorted to a separate office and given the CCQ set (with cards randomized). Children completed the intrinsic motivation inventory during the evaluation. To ensure comprehension, the inventories were orally administered, if necessary, to those children with reading difficulties.

\section{RESULTS}

All inferential statistics reported here were significant at $p<.025$, one tailed, unless otherwise reported.

Inspection of the descriptive statistics in Table 1 shows that for this sample the motivation scales show no problems with range restrictions. One of the motivation
Table 1

Sample Descriptive Statistics

\begin{tabular}{lcccc}
\hline \multicolumn{1}{c}{ Scale } & $M$ & $S D$ & Range & Alpha \\
\hline Preference for Challenge & 2.60 & .76 & $1.00-4.00$ & .77 \\
Curiosity & 2.76 & .54 & $1.67-4.00$ & .37 \\
Independent Mastery & 2.69 & .62 & $1.00-3.83$ & .62 \\
Independent Judgment & 2.18 & .67 & $1.00-3.50$ & .67 \\
Internal Criteria & 2.32 & .62 & $1.00-3.83$ & .68 \\
\hline
\end{tabular}

scales, curiosity, has a very low internal consistency. The other scales are acceptable for research purposes but may be too low for drawing strong clinical inferences.

Principal-components analyses, with orthogonal rotation in one instance, oblique in the other, were applied to the five scale scores to examine the factor structure in this sample. In both analyses, the challenge, curiosity, and independent mastery scales formed one factor, and the independent judgment and internal criteria scales formed a second factor. These results correspond well to the motivational and informational factors identified by Harter (1981). (For simplicity, Table 2 presents only the varimax-rotated factor loadings.) The two factors together accounted for $61 \%$ of the variance.

Consistent with the age trends reported by Harter (1981), chronological age in years correlated with the two scales that form the informational dimension of the intrinsic motivation scale; age correlated with independent judgment $(r=.44, p<.005$, one-tailed) and with internal criteria $(r=.59, p<.001$, one-tailed). The three motivational scales did not correlate significantly with age.

Support for the construct validity of two of the intrinsic motivation scales was obtained from correlations with the motivation composite in the CCQ. In particular, the composite correlated .30 with the preference-for-challenge scale and .25 ( $p<.05$, one-tailed) with the curiosity scale. Independent mastery, independent judgment, and internal criteria were not significantly related to the composite (respective $r s=.07,-.01$, and .19).

Academic failure was dummy coded, with children who were retained at least once forming one group (coded 1) and those never retained forming the other (coded 0 ). This classification proved to bear a meaningful relation to the curiosity $(r=-.29)$ and independent mastery $(r=-.26)$ scales ( $p<.05$, one-tailed). Thus, children who had failed a grade reported greater extrinsic motivation in their academic efforts (desiring to please the teacher rather than

Table 2

Intercorrelations Among, and Factor Loadings of, the Scales

\begin{tabular}{|c|c|c|c|c|c|c|}
\hline & \multicolumn{4}{|c|}{ Scale } & \multicolumn{2}{|c|}{ Factor } \\
\hline & 1 & 2 & 3 & 4 & I & II \\
\hline 1. Challenge & & & & & .78 & -.23 \\
\hline 2. Curiosity & $.37 *$ & & & & .76 & .07 \\
\hline 3. Mastery & .27 & .17 & & & .57 & .19 \\
\hline 4. Judgment & -.18 & -.10 & .09 & & -.17 & .85 \\
\hline 5. Criteria & .02 & .25 & .11 & $.40 *$ & .26 & .80 \\
\hline
\end{tabular}

Note $-N=50$. Eigenvalues for factors 1 and 2 were 1.63 and 1.44 , respectively. $\quad{ }^{*} p<.01$. 
to learn out of curiosity) and felt especially dependent upon their teachers in their academic efforts. Future research might address the interesting question of whether failure or self-perceptions have causal priority here. Intelligence test scores related significantly with only one of the scales used here, internal criteria $(r=.31)$. Thus, children who feel adept at judging the quality of their work are likely to have higher IQ test scores.

In order to explore the influence of other educational variables, parental educational attainment was correlated with the scales. Whereas mother's education did not correlate significantly with any of the Harter scales, father's education related to curiosity $(r=.31, p<.05$, twotailed) and possessing internal criteria for judging academic performance $(r=.42, p<.01$, two-tailed). Conceivably, these relations indicate the effects of socioeconomic status, familial educational aspirations, or both on children's academic motivations.

Finally, a multivariate analysis of variance examined the possibility of gender differences on the scales. This test yielded a sex difference that fell short of statistical significance, so it will not be discussed.

\section{DISCUSSION}

This study provides a number of impressions concerning the reliability and validity of Harter's (1981) intrinsic motivation scales. Overall, it appears that the scales have promise for the assessment of children experiencing difficulties in their academic adjustment. However, the internal consistency reliability of the scales, although acceptable for research purposes, is somewhat low for drawing inferences about individual children. Most importantly, the curiosity scale of the inventory had a very low internal consistency. Inspection of interitem correlations showed that this was due partly to one item that had low correlations with others in the scale: "Some kids work really hard to get good grades but other kids work hard because they really like to learn things." With this item removed, the scale's reliability improves, but not to an acceptable level (.54), given its low number of items. Despite this problem, the scale showed evidence of validity in this sample.

In keeping with Harter's (1981) findings, the intrinsic motivation scales formed two factors. Preference for challenge, curiosity, and indepen- dent mastery composed a motivational factor; independent judgment and internal criteria formed an informational factor. Also replicating Harter's findings, the informational factor showed marked increases with age, whereas the motivational scales did not correlate significantly with age.

The five scales had somewhat different correlates in this sample. Preference for challenge received validation in terms of its correlation with mothers' reports of their children's motivational styles on the California Child Q-Sort composite designed for this study. And, despite its low reliability, the curiosity scale also related to some extent with mothers' reports of their children's intrinsic motivation. Curiosity scores were also lower among children who had failed a grade and higher among those whose fathers had more education. Possessing internal criteria for success/failure also correlated with father's education and with measured intelligence in this sample. Thus, it appears that children who have to depend on grades and teacher reports to know how well they have done began their academic careers with some important handicapsless intelligence and probably lower socioeconomic status and/or lower educational aspirations in their families.

Taken as a whole, then, the results of this study suggest that the intrinsic motivation inventory, and particularly the preference for challenge and internal criteria scales, have promise for further clinical research. The curiosity scale also holds promise, although its reliability may vary widely from sample to sample.

\section{REFERENCES}

Amabile, T. M., Hennessey, B. A., \& Grossman, B. S. (1986). Social influences on creativity: The effect of contracted-for reward. Journal of Personality \& Social Psychology, 50, 14-23.

Block, J. H., \& Block, J. (1980). The California Child Q-Set. Palo Alto, CA: Consulting Psychologists Press.

Boggiano, A. K., \& BARReTt, M. (1985). Performance and motivational deficits of helplessness: The role of motivational orientations. Journal of Personality \& Social Psychology, 49, 1753-1761.

HARTER, S. (1978). Effectance motivation reconsidered: Toward a developmental model. Human Development, 21, 34-64.

HARTER, S. (1980). A Scale of Intrinsic versus Extrinsic Orientation in the Classroom (manual). Denver, CO: Author.

HARTER, S. (1981). A new self-report scale of intrinsic versus extrinsic orientation in the classroom: Motivational and informational components. Developmental Psychology, 17, 300-312.

Lincoln, A., \& Chazan, S. E. (1979). Perceived competence and intrinsic motivation in learning disability children. Journal of Clinical Child Psychology, 8, 213-216.

(Manuscript received for publication March 8, 1988.) 